\title{
Evaluación un proceso que mantiene los estándares de calidad
}

\author{
Evaluation a process that maintains \\ the Quality Standards
}

Recibido 05/02/2021

Evelin Yesenia Hernández Godoy

Maestría en Docencia Universitaria

Universidad de San Carlos de Gutemala

evelingodoy201043078@gmail.com

Aceptado 26/05/2021

\section{Referencia}

Hernández Godoy, E. Y. (2021). Evaluación un proceso que mantiene los estándares de calidad. Revista Docencia Universitaria, 2(2), 60-67. https://doi.org/10.46954/revistadusac.v2i2.32

\section{Resumen}

La evaluación es un proceso que requiere no solo la verificación de aspectos por mejorar, sino la toma de decisiones y por ende, la retroalimentación de los procesos obtenidos de acuerdo a los resultados. En tal sentido el artículo aborda, la evaluación como herramienta que traza planes para mejorar procesos, se trata de explicar con ello, el papel fundamental de la evaluación como parte de un mecanismo para: verificar, analizar y retroalimentar aquellos procesos que se ven desgastados o que repercuten en el logro de los estándares de calidad de educación superior. Las ideas que surgen a partir del concepto evaluación, se reflejan de manera atractiva e idónea, pero, debe tomarse en cuenta si, en realidad estas ideas son plasmadas o llevadas a la práctica de forma efectiva y pertinente, misma que se deducen de lo particular a lo general, determinando con ello, los estándares de calidad adquiridos en el estudiante de la carrera de Licenciatura en Pedagogía y Administración Educativa, del Centro Universitario de Jutiapa. 
Evidenciando con ello a través de análisis realizado, un estándar parcialmente alcanzado, lo que indica que, existen repercusiones que requieren de retroalimentación para mejorar y es esta la finalidad de la evaluación "mejorar" procesos.

\section{Abstract}

Evaluation is a process that requires not only the verification of aspects to be improved, but also decision-making and, therefore, the feedback of the processes obtained according to the results. In this sense, the article addresses evaluation as a tool that draws up plans to improve processes, it tries to explain the fundamental role of evaluation as part of a mechanism to: verify, analyze and provide feedback on those processes that are worn out or that have an impact on the achievement of higher education quality standards. The ideas that arise from the concept of evaluation are reflected in an attractive and suitable way, but it must be taken into account if, in reality, these ideas are reflected or put into practice in an effective and pertinent way, which are deduced from the particular in general, thereby determining the quality standards acquired in the student of the Bachelor's degree in Pedagogy and Educational Administration, of the Jutiapa University Center.

Evidencing with this through the analysis carried out, a partially achieved standard, which indicates that there are repercussions that require feedback to improve and this is the purpose of the evaluation to "improve" processes.

\section{Introducción}

Como afirma Mendoza (2012) la educación actual rebasa los procesos etimológicos de la didáctica, es decir, el simple arte de enseñar, sino que es un proceso que reúne un conjunto de normas, estrategias y metodologías que mejoren la enseñanza y por ende la manera de aprender del estudiante; la educación debe impulsar al estudiante a formarse como un profesional competente, capaz de asumir los nuevos retos que demanda la sociedad actual (pp. 117-119).
Palabras clave: educación, calidad, estudiante, formación, evaluación
Keywords: education, quality, student, training, evaluation 
La investigación indaga de manera profunda sobre los procesos formativos del estudiante del Centro Universitario de Jutiapa, -JUSAC-, de la carrera en Licenciatura en Pedagogía y Administración Educativa, donde se determina la situación real de estos procesos, a través de la aplicación de una serie de técnicas e instrumentos de investigación, tales como: encuesta dirigida a estudiantes por medio una escala de Likert, la observación directa, dirigida a los docentes que imparten cátedras con los estudiantes del décimo semestre de la carrera antes mencionada, aplicando como instrumento una guía de observación y por último se aplicó la técnica de grupo focal, desarrollando con ella una guía de cuestionamientos que permitieron recabar la información necesaria para determinar el nivel de logro del perfil de egreso de la carrera. El objetivo de la presente investigación es determinar la calidad de un proceso educativo, mediante el uso de la evaluación, como herramienta de medición y construcción de procesos de efectividad.

La evaluación no debe limitarse a una simple calificación, no puede concentrarse en la repetición, sino que debe de ser interactiva, entendiéndose como un desarrollo natural (Moreno, 2009). Con ello se podrá determinar la situación formativa del estudiante, verificar el logro efectivo y pertinente de las competencias inmersas en el perfil de egreso de igual forma los factores que impiden el logro del mismo.

Los hallazgos de la investigación radican en la percepción del estudiante, que finaliza la carrera universitaria, quien, a través de las técnicas e instrumentos de investigación aplicados, confirma que el logro del perfil de egreso de la carrera en Licenciatura en Pedagogía y Administración Educativa, del décimo semestre, es alcanzada en un nivel parcial, debido a factores por mejorar tales como: estrategias de aprendizaje, actualización de contenidos, rol del docente, capacitaciones a todo el personal docente y supervisión constante de los procesos formativos. Estos resultados surgen a partir primeramente de un análisis cuantitativo y se respaldan a través del análisis cualitativo, con reflexiones de cara a cara con el estudiante, fundamentando a grandes rasgos la presente investigación. 


\section{Materiales y métodos}

La investigación fue realizada en el Centro Universitario de Jutiapa, -JUSAC- específicamente en la carrera de: Licenciatura en Pedagogía y Administración Educativa, en el décimo semestre.

El proceso de investigación abarca una muestra probabilística con un total de 62 personas, distribuida a través 57 estudiantes del décimo semestre de la carrera antes mencionada y cinco docentes, abarcando con esta muestra un diseño explicativo secuencial (DEXPLIS) con un enfoque mixto establecido en las siguientes variables de investigación dependientes e independientes, siendo la primera el nivel de logro de un perfil proceso verificado a través de una evaluación y la segunda hace énfasis en las necesidades de satisfacción del estudiante, demostrando con ello cómo el proceso educativo superior se genera, es decir, si este al evaluarlo se muestra efectivo y satisfactorio.

Dentro de las técnicas e instrumentos utilizados se encuentra: la observación directa aplicada en todo el proceso de la investigación, por medio de una guía de observación, así mismo una encuesta por medio de la escala de Likert dirigida al estudiante, para determinar el nivel de satisfacción que tiene del logro del perfil de egreso de su carrera, y por último se utilizó un cuestionario dirigido aplicado a un grupo focal, donde se conoció la opinión y juicio crítico de las diferentes temáticas abordadas, por medio de un cuestionario dirigido, con la finalidad de identificar las diferentes dificultades que repercuten en logro del perfil de egreso de la carrera.

La aplicación de estas técnicas permite evaluar también la calidad de la educación superior brindada en dicho centro universitario, según De León (2016) "el sentido de la evaluación es contribuir a mejorar una realidad, para adquirir políticas y enmendar los defectos de esa realidad, eso significa que la evaluación tiene sentido si existe previamente una consciencia de que hay problemas y que necesitan ser evaluados" ( $p$. 18).

El producto de estas técnicas e instrumentos utilizados se da a través del análisis abordado, por medio de los cuales se realiza procesamiento de datos estadísticos para la representación de mayores porcentajes en las escalas de satisfacción para el estudiante, según el nivel de 
logro adquirido en el perfil de egreso, así mismo el desarrollo de transcripciones para la interpretación de grupos focales. Reflejando por medio de ello el logro parcial del perfil de egreso de la carrera. Y es que este proceso se vuelve evaluativo, debido a que se obtuvo información real y profunda desde el punto de vista del estudiante y la perspectiva del qué hacer del docente, donde ambas perspectivas de muestran que hay varios factores por mejorar para que las competencias establecidas en el perfil de egreso sean alcanzadas en un nivel de satisfacción efectivo y por ende el proceso formativo se convierta en educación de calidad.

\section{Resultados}

Los estándares de calidad en Educación Superior del Centro Universitario de Jutiapa, JUSAC, de la carrera de Licenciatura en Pedagogía y Administración Educativa, deben verificarse de forma constante, cuando esto sucede, el proceso permite que las deficiencias encontradas sean mejoradas y que estas debilidades se conviertan en fortalezas; principalmente la idea de evaluar en este caso un plan de estudios es, reconocer sus fallas y también aciertos, claro está que en este proceso no solamente se verificarán errores, sino también todos aquellos procedimientos que han contribuido en gran medida a la calidad educativa, y de estos últimos fortalecerlos para mantener la excelencia formativa (Rodríguez Pérez, 2017).

Dentro de los resultados de la investigación se determinó, a través de la evaluación el nivel de satisfacción del perfil de egreso de la carrera de Licenciatura en Pedagogía y Administración Educativa, estableciendo a través de un escenario cuantitativo y cualitativo que el nivel de satisfacción para cada estudiante se da de forma parcial, siendo el punto de partida para la toma de decisiones, para que los procesos formativos se brinden con calidad. Así mismo, conocer las deficiencias que repercuten en el logro de las competencias de cada estudiante, siendo estas la pauta para la toma de decisiones en pro de mejoras para la institución y por ende para el estudiante.

Los datos estadísticos se demuestran en gráficas que resumen de manera cuantitativa el dominio de las competencias establecidas en el perfil de egreso, indicando a través de ellos cuál es el nivel de logro de este perfil en el estudiante. Observa en la figura No. 1 los resultados obtenidos. 


\section{Figura No. 1}

\section{Domino de las habilidades profesionales}

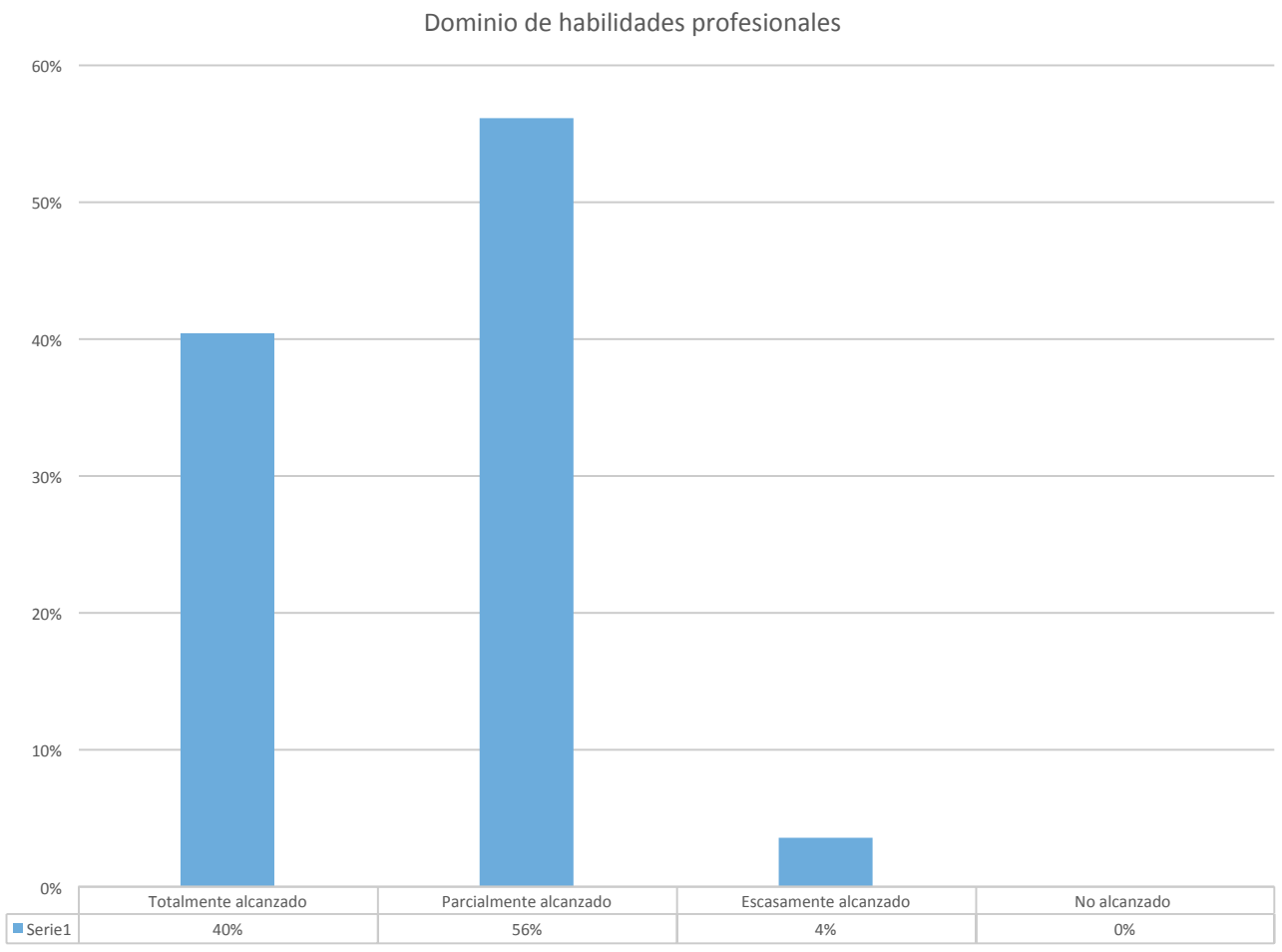

Fuente: propia, tomada de la base de datos del trabajo de campo (2019).

Los resultados obtenidos surgen a partir de los indicadores establecidos en un cuestionario de escalamiento Likert, siendo los indicadores: totalmente alcanzado, parcialmente alcanzado, escasamente alcanzado y no alcanzado, tomando en cuenta que la población total es de 57 personas, siendo los resultados los siguientes: en un $40 \%$ se refleja que el dominio de las habilidades profesionales se manifiesta de forma total, el $56 \%$ indica que el dominio de estas habilidades se manifiesta de forma parcial, el $4 \%$ en un logro escaso y el $0 \%$ no alcanzado. Siendo entonces, el $56 \%$ el porcentaje con mayor incidencia en el logro de estas competencias, lo que indica que existen factores que repercuten en el logro total de las habilidades como profesionales.

\section{Discusión}

El mayor porcentaje se refleja en una escala parcial, siendo el más alto un $56 \%$, de la misma forma en que se reflejan el resto de porcentajes. Justificando y complementando este resultado con la 
entrevista dirigida en el grupo focal, donde el estudiante manifiesta inconformidades y satisfacciones en su formación profesional; dentro de las inconformidades que regulan el nivel de logro total del perfil a un nivel parcial, se encuentra el tiempo, la labor docente, las estrategias y metodologías utilizadas para la formación del estudiante, en tan sentido estas dificultades son las que generan la problemática de investigación.

Dicha investigación requiere para dar solución a la situación problema, una evaluación constante del proceso global educativo de cada estudiante, haciendo énfasis en los procesos cognitivos y administrativos que imperan en dicha institución y de esta manera asegurar el logro del perfil del egresado.

\section{Referencias bibliográficas}

De León, M. (2016). Evaluación del proceso de formación del docente en el Centro Universitario de El Progreso. Alemania. Recuperado el mayo de 2019

Mendoza, A. C. (2012). Educar en un mundo Sociovirutal, Pedagogía Crítica en el siglo XXI. Guatemala : Universitaria.

Moreno, T. (2009). La evaluación del aprendizaje en la universidad: tensiones, contradicciones y desafíos. Revista mexicana de investigación educativa, vol 14 No. 41.

Rodríguez Pérez, I. (2017). La calidad de la educación superior y la reeestrucutración del Programa de Tutoría. Revista Iberoaméricana para la Investigación y el Desarrollo Educativo., 8(15), 1-20. 


\section{Sobre la autora}

Es Licenciada en Pedagogía y Administración Educativa y actualmente, estudiante con cierre de pensum en el año 2019 de la Facultad de Humanidades, en la Maestría en Docencia Universitaria del campus central USAC, tiene experiencia en investigación de 8 años, desde la elaboración de proyectos, EPS, tesis y artículos de investigación.

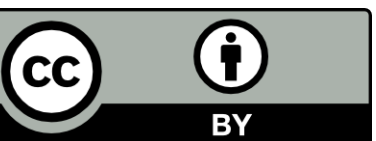

Este texto está protegido por una licencia Creative Commons 4.0.

Usted es libre para compartir, copiar y redistribuir el material en cualquier medio o formato y adaptar el documento, remezclar, transformar y crear a partir del material para cualquier propósito, incluso comercialmente, siempre que cumpla la condición de atribución: usted debe reconocer el crédito de una obra de manera adecuada, proporcionar un enlace a la licencia, e indicar si se han realizado cambios. Puede hacerlo en cualquier forma razonable, pero no de forma tal que sugiera que tiene el apoyo del licenciante o lo recibe por el uso que hace. 